Diabetologe 2021 · 17:36-41 https://doi.org/10.1007/s11428-020-00715-7 Angenommen: 17. Dezember 2020 Online publiziert: 11. Januar 2021 (c) Springer Medizin Verlag $\mathrm{GmbH}$, ein Teil von Springer Nature 2021

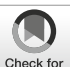

Stefan R. Bornstein ' Baptist Gallwitz ${ }^{2}$ Monika Kellerer ${ }^{3}$ Barbara Ludwig' Dirk Müller-Wieland ${ }^{4} \cdot$ Andreas Neu $^{5} \cdot$ Hans-Martin Reuter ${ }^{6} \cdot$ Michael Roden $^{7}$. Jochen Seufert ${ }^{8}$

${ }^{1}$ Medizinische Klinik und Poliklinik III, Universitätsklinikum Carl Gustav Carus, Dresden, Deutschland

${ }^{2}$ Department Innere Medizin, Abt. IV, Universitätsklinikum Tübingen, Tübingen, Deutschland

${ }^{3}$ Zentrum für Innere Medizin I, Marienhospital, Stuttgart, Deutschland

${ }^{4}$ Medizinische Klinik I, Universitätsklinikum der RWTH Aachen, Aachen, Deutschland

${ }^{5}$ Klinik für Kinder- und Jugendmedizin, Universitätsklinikum Tübingen, Tübingen, Deutschland

${ }^{6}$ Diabetologische Schwerpunktpraxis, Gemeinschaftspraxis für Innere Medizin, Jena, Deutschland

${ }^{7}$ Medizinische Klinik, Klinik für Endokrinologie und Diabetologie, Universitätsklinikum Düsseldorf, Düsseldorf, Deutschland

${ }^{8}$ Klinik für Innere Medizin II, Universitätsklinikum Freiburg, Freiburg, Deutschland

\title{
Praktische Empfehlungen der Deutschen Diabetes Gesellschaft zum Diabetesmanagement bei Patientinnen und Patienten mit einer COVID-19-Erkrankung
}

Risiko für einen schweren Krankheitsverlauf einschließlich ,acute respiratory distress syndrome" (ARDS) und Multiorganversagen bei COVID-19-Erkrankung zu haben. Ob es sich dabei um eine bloße Assoziation oder um kausale Zusammenhänge handelt, ist für das neuartige Coronavirus SARS-CoV-2 derzeit nicht geklärt. Signalwege über das Angiotensinkonversionsenzym 2 (ACE2) könnten einen spezifischen Einfluss haben, da das Coronavirus-Spike-Protein den ACE2Rezeptor für den Zelleintritt nutzt [1]. ACE2 bewirkt in der Lunge eine Degradierung von Angiotensin $2 \mathrm{zu}$ Angiotensin 1-7, das in Zell- und Tiermodellen antiinflammatorische und antifibrotische Effekte zeigte [2]. Einzelne Arbeiten deuten zudem auf eine mögliche Beziehung zwischen Coronavirusinfektionen, ACE2-Expression und Glukosestoffwechsel hin [3-6]. Gegenwärtig gibt es keine klinischen Daten, aus denen man einen solchen Zusammenhang bei einer COVID-19-Erkrankung ableiten könnte.

Aufgrund der Häufigkeit von Diabetes mellitus als Komorbidität bei COVID19 wollen wir im Folgenden nochmals auf einige prinzipielle Handlungsempfehlungen bei Diabetes und COVID-19 hinweisen.

Die vorliegenden Empfehlungen beziehen sich auf Erwachsene. Für Kinder und Jugendliche gelten die Behandlungsstrategien und Dosisangaben, die in der DDG-Leitlinie Diagnostik, Therapie und Verlaufskontrolle des Diabetes mellitus im Kindes- und Jugendalter (http://www.deutsche-diabetesgesellschaft.de/fileadmin/Redakteur/ Leitlinien/Evidenzbasierte_Leitlinien/ DM_im_Kinder-_und_Jugendalter_ 20151206.pdf) festgelegt sind.

Wir verweisen darüber hinaus auf die Praxisempfehlungen und Leitlinien der DDG: https://www.deutsche-diabetesgesellschaft.de/leitlinien/praxisempfeh lungen.

Patienten mit Diabetes mellitus und blandem Verlauf von COVID-19, die nicht stationär therapiert werden müssen, sollten im telefonischen Kontakt mit der diabetologischen Schwerpunktpraxis bzw. ambulanten spezialisierten Versorgungseinrichtung stehen, insbesondere bei Insulintherapie oder mit potenziell 
Praxisempfehlungen der DDG

COVID-19 und metabolische Erkrankungen

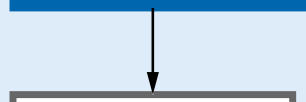

Prävention von

Infektion bei

Menschen mit

bestehendem

Diabetes

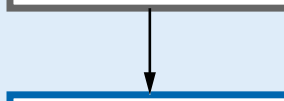

Notwendigkeit zur

Optimierung der

Glykämiekontrolle

(Plasmaglukose,

$\mathrm{HbA1c}$ ) und RR-

Monitoring
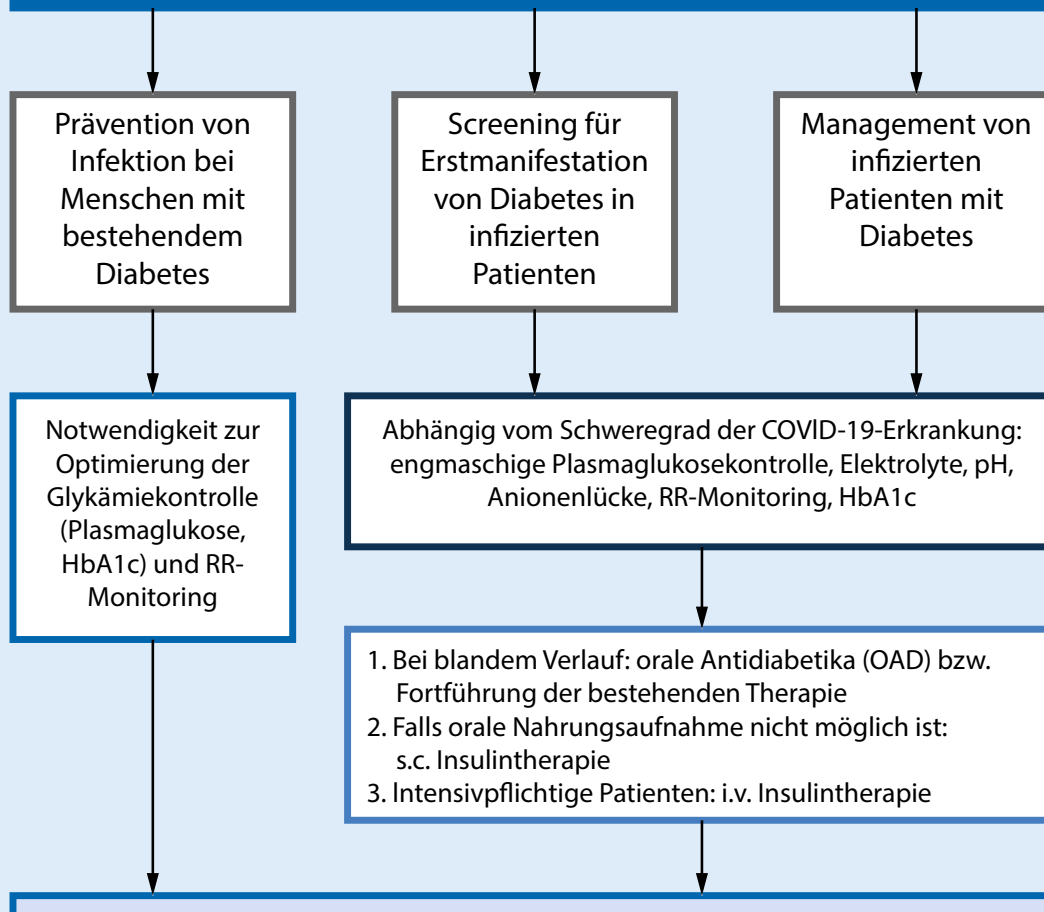

Abhängig vom Schweregrad der COVID-19-Erkrankung: engmaschige Plasmaglukosekontrolle, Elektrolyte, $\mathrm{pH}$ Anionenlücke, RR-Monitoring, $\mathrm{HbA} 1 \mathrm{c}$

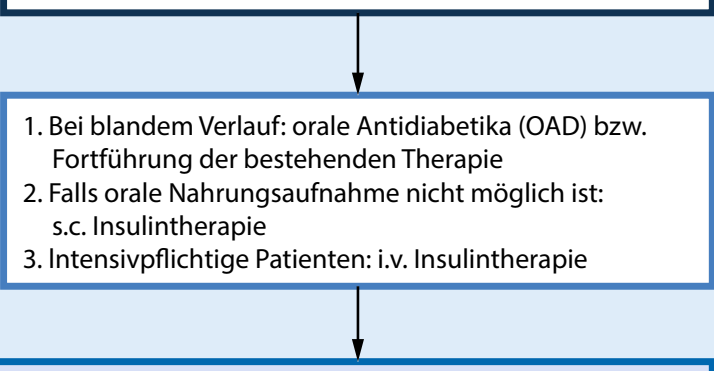

Therapieziele:

bei nicht-intensivpflichtigen Patienten: idealerweise 70-180 mg/dl; 3,9-10 mmol/dl unter Vermeidung von Hypoglykämien,

HbA1c-Zielwert $<7,5 \%$ entsprechend den Praxisempfehlungen der DOG bei intensivpflichtigen Patienten: $140-180 \mathrm{mg} / \mathrm{dl} ; 7,8-10 \mathrm{mmol} / \mathrm{dl}^{\mathrm{a}}$ $\mathrm{RR}<135 / 85 \mathrm{mmHg}$

Beachte mögliche antidiabetische Effekte von:

- Hydroxychloroquin

- Camostat

- rekombinante ACE 2

Beachte mögliche diabetogene Effekte von:

- Glukokortikoiden

- antiviraler Medikation

Abb. $1 \Delta$ Flussdiagramm Diabetesscreening und Management von Patienten mit COVID-19. Daten nach [6]. ${ }^{\text {II }}$ den meisten Studien werden Blutglukosewerte zwischen 140 und $180 \mathrm{mg} / \mathrm{dl}$ (7,8 und $10 \mathrm{mmol} / \mathrm{l}$ ) als ausreichend sicher angesehen, um einerseits hyperglykämiebedingte Komplikationen zu vermeiden und um andererseits auch die Hypoglykämierate möglichst gering zu halten. Es wird empfohlen, eine antihyperglykämische Therapie bei Blutglukosewerten ab $180 \mathrm{mg} / \mathrm{dl}(10 \mathrm{mmol} / \mathrm{l})$ zu beginnen bzw. zu intensivieren. Bei Werten unter $110 \mathrm{mg} / \mathrm{dl}(6,1 \mathrm{mmol} / \mathrm{l})$ sollen hingegen blutglukoseerhöhende Maßnahmen ergriffen werden. Diese Empfehlung zur Blutglukoseeinstellung auf Intensivstationen findet sich auch in internationalen Leitlinien [7] sowie in der Leitlinie zur Prävention, Diagnose, Therapie und Nachsorge der Sepsis wieder [8] problematischer OAD-Therapie. Frauen mit einem Gestationsdiabetes, Menschen mit Typ-1-Diabetes und Patienten mit Komorbiditäten und COVID-19Erkrankung sollten telefonisch oder telemedizinisch (z. B. per Videosprechstunde) engmaschig kontrolliert und begleitet werden.

Patienten mit vorbestehendem Diabetes mellitus sollten als Primärpräventionsmaßnahme für jegliche Infektion einschließlich SARS-CoV-2 ihre metabolische Kontrolle optimieren.

Infizierte Patienten ohne vorbekannten Diabetes mellitus sollten durch Glukosemonitoring oder $\mathrm{HbA}_{1 \mathrm{c}}$-WertBestimmung bezüglich einer Neumanifestation getestet werden.

Bei Diabetes und schweren Verläufen von COVID-19, insbesondere solchen mit Krankenhausaufenthalt, wird initial und passager vielfach Insulin zum Einsatz kommen, da hier am wenigsten Komplikationen (z.B. Laktatazidose, Ketoazidose) und Medikamenteninteraktionen (u. a. auch mit experimenteller antiviraler Therapie wie Hydroxychloroquin, Lopinavir/Ritonavir, Remdesivir usw.) zu erwarten sind. Andere antidiabetische Therapieoptionen sollten kritisch hinsichtlich sog. „sick day rules“ überprüft und angepasst werden. Dies gilt insbesondere für SGLT-2-Inhibitoren zur Vermeidung einer atypischen Ketoazidose und für Metformin hinsichtlich einer Laktatazidose. Sulfonylharnstoffe sollten wegen der Gefahr von Hypoglykämien bei Kumulation aufgrund einer (transienten) Niereninsuffizienz ebenfalls pausiert werden.

\section{Praktische Empfehlungen zum Umgang mit SGLT- 2-Inhibitoren bei akuten Infektionskrankheiten}

Pausieren bei

- Fieber $>38,5^{\circ} \mathrm{C}$

- eingeschränkter Nahrungs- oder

Flüssigkeitsaufnahme und/oder

- Insulinmangel (dabei Überprüfung des Säure-Basen-Haushalts, der Ketone im Serum und der Nierenfunktion). 
Wiederaufnahme der Therapie erst bei

- Fieberfreiheit,

- ausreichender Flüssigkeits- und Nahrungsaufnahme,

- $\mathrm{eGFR}>60 \mathrm{ml} / \mathrm{min} / 1,73 \mathrm{~kg} / \mathrm{m}^{2}$,

- ausgeglichenem Säure-Basen-Haushalt und

- nicht erhöhten Ketonen im Serum.

Bei einer vorbestehenden Dapagliflozintherapie bei Typ-1-Diabetes soll diese generell bei akuten Infektionskrankheiten - einschließlich COVID-19 - pausiert werden. Ein Wiederansetzen sollte erst nach vollständiger Genesung und nach vorheriger Evaluation durch einen Diabetologen erfolgen.

\section{Praktische Empfehlungen zum Umgang mit Metformin bei akuten Infektionskrankheiten}

- Pausieren bei Fieber $>38,5^{\circ} \mathrm{C}$

- Engmaschige Überprüfung der Nierenfunktion: falls eGFR

$30-60 \mathrm{ml} / \mathrm{min} / 1,73 \mathrm{~kg} / \mathrm{m}^{2}$ reduzierte Dosis von max. $1000 \mathrm{mg} / \mathrm{Tag}$ möglich, bei eGFR $<30 \mathrm{ml} \mathrm{ml} / \mathrm{min} / 1,73 \mathrm{~kg} / \mathrm{m}^{2}$ ist Metformin kontraindiziert!

- Überprüfung des Säure-Basen-Haushalts und des Laktats im Serum bei vorheriger Metformineinnahme und fortgeschrittener Niereninsuffizienz oder passagerem Nierenversagen

- Wiederaufnahme der Therapie erst bei

- Fieberfreiheit,

- ausreichender Flüssigkeits- und Nahrungsaufnahme,

- eGFR > $30 \mathrm{ml} / \mathrm{min} / 1,73 \mathrm{~kg} / \mathrm{m}^{2}$ und

- Ausschluss einer Hypoxämie.

DPP-4-Inhibitoren müssen während der COVID-19-Erkrankung nicht pausiert werden, wenn die orale Medikamenteneinnahme möglich ist und eine ausreichende antihyperglykämische Wirkung zu erwarten ist. Der Einsatz kann gemäß Fachinformation fortgeführt werden.

Pioglitazon sollte während der COVID-19-Erkrankung pausiert werden, da im Rahmen der Infektion mit SARS-CoV-2 sowohl eine Niereninsuffizienz als auch eine Herzinsuffizienz mit der Gefahr einer vermehrten

Diabetologe 2021 · 17:36-41 https://doi.org/10.1007/s11428-020-00715-7

(c) Springer Medizin Verlag GmbH, ein Teil von Springer Nature 2021

S. R. Bornstein · B. Gallwitz · M. Kellerer · B. Ludwig · D. Müller-Wieland · A. Neu • H.-M. Reuter · M. Roden $\cdot$ J. Seufert

\section{Praktische Empfehlungen der Deutschen Diabetes Gesellschaft zum Diabetesmanagement bei Patientinnen und Patienten mit einer COVID-19-Erkrankung}

\section{Zusammenfassung}

Bei Menschen mit einer Infektion mit dem neuartigen Coronavirus SARS-CoV-2 und einer COVID-19-Erkrankung sind Diabetes mellitus und kardiovaskuläre Erkrankungen sehr häufige Komorbiditäten. Patienten mit Diabetes und Begleit- beziehungsweise Folgeerkrankungen scheinen ein erhöhtes Risiko für einen schweren Krankheitsverlauf bei COVID-19 einschließlich ARDS und Multiorganversagen zu haben. Die genauen Zusammenhänge zwischen Diabetes und
COVID-19-Krankheitsverlauf sind noch nicht vollständig geklärt. Die DDG hat daher prinzipielle Handlungsempfehlungen zur Diabetestherapie bei Diabetes mellitus und COVID-19-Erkrankung zusammengefasst.

Schlüsselwörter

Diabetestherapie - COVID-19-Erkrankung . SARS-CoV-2-Infektion · Typ-1-Diabetes · Typ2-Diabetes

\section{Practical recommendations of the German Diabetes Society for the management of diabetes in patients with COVID-19}

\section{Abstract}

In subjects with an infection with the novel corona virus SARS-CoV-2 and COVID-19 disease, diabetes mellitus and cardiovascular diseases are highly prevalent comorbidities. Diabetes patients with comorbidities and/or diabetes complications seem to have a higher risk for a severe course of the COVID-19 disease including the development of ARDS or multiorgan failure. The exact interrelations between diabetes and the individual clinical course of COVID-19 are not completely understood at this time. The German Diabetes Association (DDG) summarizes basic principle practice recommendations for the treatment of patients with diabetes and concomitant COVID-19 disease.

Keywords Diabetes therapy · COVID 19 disease - SARS CoV 2 infection - Type 1 diabetes - Type 2 diabetes
Flüssigkeitsretention und -einlagerung bestehen.

GLP-1-Rezeptor-Agonisten können formal gemäß Fachinformation und unter Berücksichtigung der Nierenfunktion weiter eingesetzt werden. Bei schweren Verläufen der COVID-19-Erkrankung ist eine Pausierung zugunsten einer Insulintherapie zu diskutieren.

Bei schweren Verlaufsformen der COVID-19-Erkrankung sollte bei Notwendigkeit einer intensivmedizinischen Therapie die Indikation zur i.v. Insulintherapie großzügig gestellt werden, da durch sie meist eine optimale Steuerbarkeit der Glykämiekontrolle gewährleistet ist und Resorptionsstörungen aus dem subkutanen Gewebe umgangen werden können.

\section{Antihypertensive Therapie}

Der Zielblutdruck ist $<135 / 80 \mathrm{~mm} \mathrm{Hg}$ unter antihypertensiver Therapie.

Da das SARS-CoV-2-Virus über den ACE2-Rezeptor in die Zellen eindringt, wurde ein möglicher Zusammenhang zwischen der Einnahme von RAS-Blockern (dadurch Erhöhung der ACE2Expression?) und der Entwicklung einer schweren COVID-19-Erkrankung einschließlich ARDS diskutiert [9, 10]. Für solche hypothetischen Überlegungen liegt jedoch keine klinische Evidenz vor, sodass - auch aufgrund der wissenschaftlich belegten positiven Effekte von RAS-Blockern auf das CV- und renale System - derzeit kein Absetzen einer bestehenden RAS-Inhibitoren-Therapie empfohlen wird. Entsprechend aktuel- 


\begin{tabular}{llll}
\hline Tab. 1 & Blutglukosezielwerte & & \\
\hline Zu niedrig & In einzelnen Patienten & Zielwert & Zu hoch \\
\hline$<110 \mathrm{mg} / \mathrm{dl}$ & $110 \mathrm{mg} / \mathrm{dl}$ & $140 \mathrm{mg} / \mathrm{dl}$ & $>180 \mathrm{mg} / \mathrm{dl}$ \\
$(6,1 \mathrm{mmol} / \mathrm{l})$ & $(6,1 \mathrm{mmol} / \mathrm{l})$ & $(7,8 \mathrm{mmol} / \mathrm{l})$ & $(10 \mathrm{mmol} / \mathrm{l})$ \\
& $140 \mathrm{mg} / \mathrm{dl}$ & $180 \mathrm{mg} / \mathrm{dl}$ & \\
& $(7,8 \mathrm{mmol} / \mathrm{l})$ & $(10 \mathrm{mmol} / \mathrm{l})$ &
\end{tabular}

Tab. 2 Aktuelle Blutglukosewerte $<75 \mathrm{mg} / \mathrm{dl}(4,2 \mathrm{mmol} / \mathrm{l})$

\begin{tabular}{|c|c|}
\hline Blutglukose & Maßnahmen \\
\hline $\begin{array}{l}<50 \mathrm{mg} / \mathrm{dl} \\
(2,8 \mathrm{mmol} / \mathrm{l})\end{array}$ & $\begin{array}{l}\text { Stopp Insulinperfusor, } 250 \mathrm{ml} \text { Glukose } 10 \%(25 \mathrm{~g}) \text { i.v., BG-Kontrolle nach } \\
15 \mathrm{~min} \\
\text { BG }>120 \mathrm{mg}(6,7 \mathrm{mmol} / \mathrm{l}) \text { nach } 1 \mathrm{~h} \text { : erneuter Start des Insulinperfusors mit } \\
50 \% \text { der vorher gelaufenen Infusionsrate }\end{array}$ \\
\hline $\begin{array}{l}50-74 \mathrm{mg} / \mathrm{dl} \\
(2,8-4,1 \mathrm{mmol} / \mathrm{l})\end{array}$ & $\begin{array}{l}\text { Stopp Insulinperfusor, } 120 \mathrm{ml} \text { Glukose } 10 \%(12 \mathrm{~g}) \text { i.v., BG-Kontrolle nach } \\
15 \mathrm{~min} \\
\text { BG > } 120 \mathrm{mg}(6,7 \mathrm{mmol} / \mathrm{l}) \text { nach } 1 \mathrm{~h} \text { : erneuter Start des Insulinperfusors mit } \\
75 \% \text { der vorher gelaufenen Infusionsrate }\end{array}$ \\
\hline
\end{tabular}

ler Evidenz sollten Patienten somit das bestehende antihypertensive Behandlungsregime beibehalten, einschließlich ACE-Hemmer und Angiotensinrezeptorblocker $[11,12]$.

\section{Statintherapie}

Eine ggf. bestehende Therapie mit Statinen wird beibehalten. Im Rahmen der COVID-19-Erkrankung finden sich häufig auch erhöhte CK-Werte. Es sollte deshalb auch die CK im Serum bei bestehender Statintherapie (insbesondere bei schwereren COVID-19-Verläufen) bestimmt werden. Bei CK-Anstieg sollte das Statin pausiert werden.

Wichtig ist die Beachtung der potenziell antidiabetischen und diabetogenen Effekte von Medikamenten, die bei COVID-19-Erkrankung zum Einsatz kommen könnten (• Abb. 1).

\section{Berücksichtigung von Medikamenteninteraktionen}

Insbesondere beim (experimentellen) Einsatz von antiviraler Begleitmedikation (Hydroxychloroquin, Lopinavir/Ritonavir, Remdesivir, Tocilizumab) sollten Medikamenteninteraktionen mit antidiabetischen Substanzen berücksichtigt werden. Die DDG empfiehlt dazu auch, sich über die COVID-19-Wechselwirkungsplattform der „University of Liverpool“ zu informieren: https://www. covid19-druginteractions.org/.
Blutglukosemanagement bei i.v. Insulintherapie auf Intensivoder Überwachungsstationen

Insulinperfusortherapie modifiziert nach Yale-Protokoll, Daten nach [13]

\section{Anwendung}

- Lebensbedrohlich Erkrankte auf der Intensivstation mit Hyperglykämie, nicht bei hyperosmolarem Koma oder bei diabetischer Ketoazidose oder bei Blutglukose (BG) $>500 \mathrm{mg} / \mathrm{dl}$ $(27,7 \mathrm{mmol} / \mathrm{l})$

- Nicht anwendbar bei Patienten, die orale Kost aufnehmen

Füllung des Insulinperfusors: $1 \mathrm{IE} / \mathrm{ml}$ Normalinsulin, z. B. $50 \mathrm{IE}$ ad $50 \mathrm{ml} \mathrm{NaCl}$ $0,9 \%$

Blutglukosezielwerte (• Tab. 1):

- Zielwert: $140 \mathrm{mg} / \mathrm{dl}(7,8 \mathrm{mmol} / \mathrm{l})$ bis $180 \mathrm{mg} / \mathrm{dl}(10 \mathrm{mmol} / \mathrm{l})$

- Beginn mit Insulin: Blutglukose $>180 \mathrm{mg} / \mathrm{dl}(10 \mathrm{mmol} / \mathrm{l})$

- Niedrige Zielwerte im Einzelfall sinnvoll

\section{Durchführung}

Initial erhält der Patient einen Bolus. Die Einheitenzahl ist identisch mit der Startdosis der ersten Stunde: Beispiel: Blutglukose $=271 \mathrm{mg} / \mathrm{dl}$ $(15,0 \mathrm{mmol} / \mathrm{l}) / 100=2,7$ Einheiten als Bolus und 2,7 Einheiten über die erste Stunde.
Benötigt werden 3 Werte:

- Aktuelle Blutglukose (BG)

- Blutglukosewert vor $1 \mathrm{~h}$, Differenz zum aktuellen Blutglukosewert

- Perfusorinfusionsrate

Bei Blutglukosewerten $\quad<75 \mathrm{mg} / \mathrm{dl}$ $(4,2 \mathrm{mmol} / \mathrm{l})$ soll der Anweisung aus - Tab. 2 gefolgt werden; bei Blutglukosewerten $>75 \mathrm{mg} / \mathrm{dl}(4,2 \mathrm{mmol} / \mathrm{l})$ der aus - Abb. 2.

Kontrollintervalle: Stündlich bis 3-mal im Zielbereich $\rightarrow$ dann alle $2 \mathrm{~h}$ bis Blutglukose über $12 \mathrm{~h}$ stabil $\rightarrow$ dann alle $4 \mathrm{~h}$

\section{Umstellung von Insulinperfusor auf s.c. Insulintherapie}

Die Insulintherapie muss $1-2 \mathrm{~h}$ überlappend erfolgen. Dabei können die Patienten mit vorbestehender Insulintherapie diese wieder aufnehmen.

Die anderen Patienten sollten eine intensivierte Insulintherapie beginnen:

- Initiale Umstellung auf subkutane Insulingabe, Gesamtdosis: 0,6 E/kg/Tag (Beispiel: $80 \mathrm{~kg}=48$ Einheiten)

- Je $25 \%$ morgens und zur Nacht als NPH-Insulin (Beispiel: je 12 Einheiten) oder

- $45 \%$ eines lang wirkenden Insulinanalogons zu einem beliebigen, aber festen Zeitpunkt (Beispiel: 22 Einheiten)

Zu den Mahlzeiten: kurz wirkendes Insulinanalogon oder Normalinsulin im Verhältnis 2,0-1,0-1,5 E/KHE, wenn das Zählen der KHE auf der Station möglich ist

Ansonsten die Gesamtdosis dividiert durch $10=$ Mittagsdosis (Beispiel 5 Einheiten), morgens die doppelte Dosis (Beispiel 10 Einheiten) und abends die 1,5fache Dosis (Beispiel 8 Einheiten)

Zusätzlich jeweils ein Korrekturinsulin mit Angabe des Zielwerts (Beispiel $140 \mathrm{mg} / \mathrm{dl}$ [7,8 $\mathrm{mmol} / \mathrm{l}])$ und einer Korrekturregel (Beispiel 1 Einheit Insulin senkt den Blutglukosewert um ca. $40 \mathrm{mg} / \mathrm{dl}$ [ $2 \mathrm{mmol} / \mathrm{l}]$ ). 


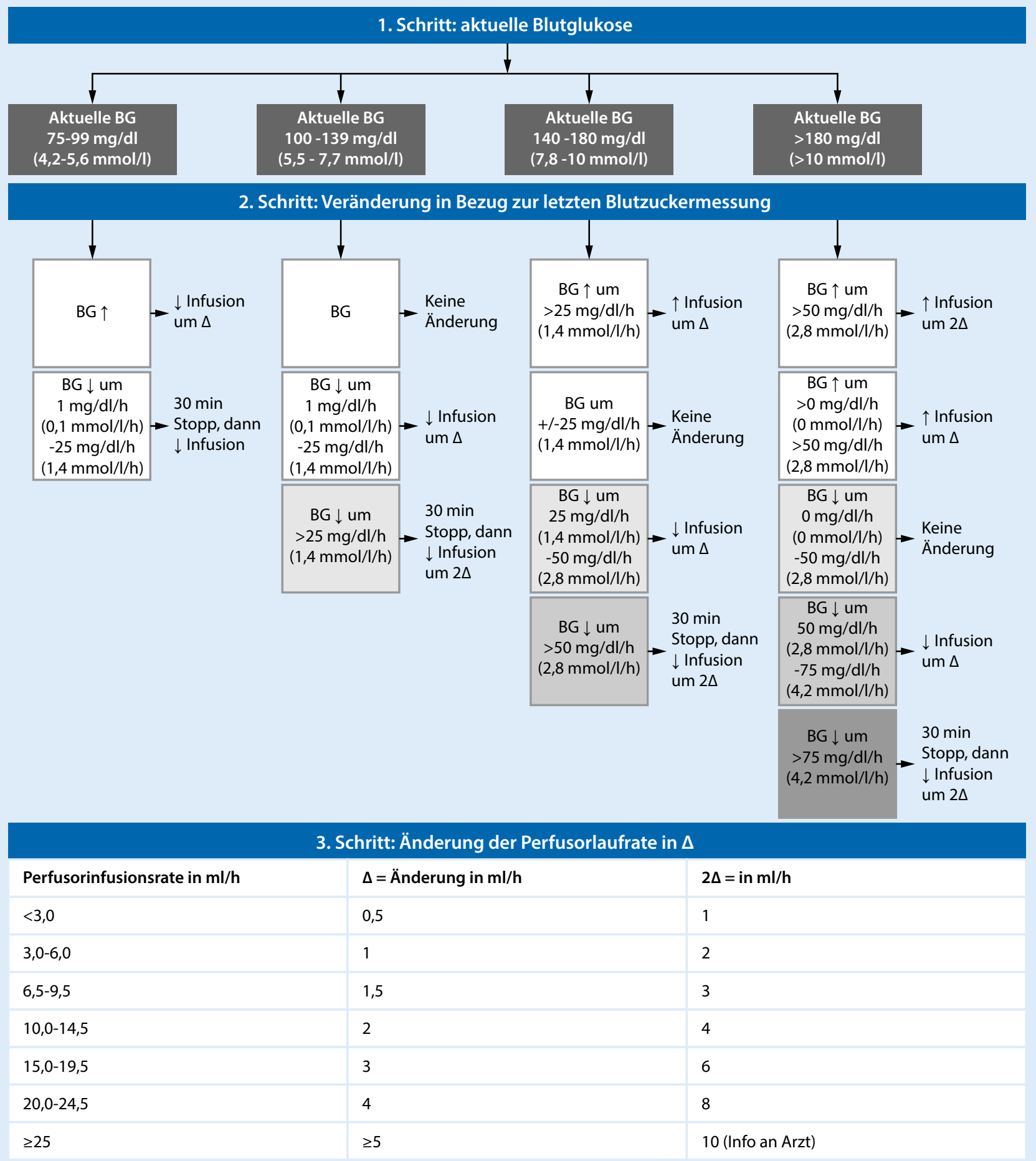

Abb. 2 ム Protokoll zur i.v. Insulintherapie auf Intensivstation 


\section{Korrespondenzadresse}

Prof. Dr. med. Monika Kellerer

Zentrum für Innere Medizin I, Marienhospital Böheimstr. 37, 70199 Stuttgart, Deutschland monika.kellerer@vinzenz.de

\section{Einhaltung ethischer Richtlinien}

Interessenkonflikt. S.R. Bornstein, B. Gallwitz, M. Kel lerer, B. Ludwig, D. Müller-Wieland, A. Neu, H.-M. Reuter, M. Roden und J. Seufert geben an, dass kein Interessenkonflikt besteht

\section{Literatur}

1. Hoffmann $M$, Kleine-Weber $H$, Schroeder $\mathrm{S}$ et a (2020) SARS-CoV-2 cell entry depends on ACE2 and TMPRSS 2 and is blocked by a clinically proven protease inhibitor. Cell 181:271-280.e8. https:// doi.org/10.1016/j.cell.2020.02.052

2. Simões e Silva AC, Silveira KD, Ferreira AJ et al (2013) ACE2, angiotensin-(1-7) and Mas receptor axis in inflammation and fibrosis. Br J Pharmacol 169:477-492. https://doi.org/10.1111/bph.12159

3. Bindom SM, Lazartigues E (2009) The sweeter side of ACE2: physiological evidence for a role in diabetes. Mol Cell Endocrinol 302:193-202

4. Roca-Ho H, Riera M, Palau V et al (2017) Characterization of ACE and ACE2 expression within different organs of the NOD mouse. Int J Mol Sci 18:E563. https://doi.org/10.3390/ ijms 18030563

5. Yang JK, Lin SS, Ji XJ et al (2010) Binding of SARS coronavirus to its receptor damages islets and causes acute diabetes. Acta Diabetol 47:193-199

6. Bornstein SR, Dalan R, Hopkins D et al (2020) Endocrine and metabolic link of coronavirus infection. Nat Rev Endocrinol 16:297-298. https:// doi.org/10.1038/s41574-020-0353-9

7. Kavanagh BP, McCowen KC (2010) Clinical practice. Glycemic control in the ICU. N Engl J Med 363:2540-2546

8. Deutsche Sepsis-Gesellschafte. V. (DSG), Deutsche Interdisziplinäre Vereinigung für Intensiv- und Notfallmedizin (DIVI) (2010) Prävention, Diagnose, Therapie und Nachsorge der Sepsis. 1. Revision der S2k Leitlinie. https://sepsis-hilfe.org/fileadmin/ user_upload/pdf/leitlinien_s-2k_sepsis.pdf

9. Zheng YY, Ma YT, Zhang JY et al (2020) COVID19 and the cardiovascular system. Nat Rev Cardiol 17:259-260

10. Fang L, Karakiulakis G, Roth M (2020) Are patients with hypertension and diabetes mellitus at increased risk for Covid-19 infection? Lancet Respir Med 8:E21. https://doi.org/10.1016/S22132600(20)30116-8

11. Wendt R, Beige J, Lübbert C (2020) Covid-19: Der Einfluss der Antihypertonika. Dtsch Arztebl 117:A-664-B-565

12. Danser AHJ, Epstein M, Batlle D (2020) Reninangiotensin system blockers and the COVID-19 pandemic. Hypertension 75:1382-1385

13. Shetty S, Inzucchi S, Goldberg P et al (2012) Adapting to the new consensus guidelines for managing hyperglycemia during critical illness: the updated Yale insulin infusion protocol. Endocr Pract 18:363-370

\section{Studie untersucht Wirkung von Teplizumab auf den Erhalt der Insulinproduktion in der Bauchspeicheldrüse bei Kindern}

Der Antikörper Teplizumab kann bei Personen mit erhöhtem familiärem Risiko die Entwicklung eines Typ-1-Diabetes verzögern Darauf deuten Untersuchungen hin. Wie Teplizumab bei Kindern und Jugendlichen mit einem neu diagnostizierten Typ-1-Diabetes wirkt, soll nun in der PROTECT-Studie untersucht werden.

Typ-1-Diabetes (T1D) ist die häufigste Stoffwechsel- und Autoimmunerkrankung bei Kindern. In Deutschland leben circa 373.000 Menschen mit einem Typ-1-Diabetes und jedes Jahr erkranken etwa 3.100 Kinder und Jugendliche zwischen 0 und 17 Jahren neu daran. Menschen mit Typ-1-Diabetes sind ihr Leben lang auf künstliches Insulin angewiesen. Genetische Faktoren erhöhen das Risiko der Erkrankung. Bisher sind mehr als 50 krankheitsrelevante Genorte bekannt, die alle einen Einfluss auf die Immunantwort zu haben scheinen.

\section{Schutz für Betazellen}

Um den Ausbruch der Erkrankung zu verhindern bzw. die Folgen der Erkrankung zu mildern, suchen Wissenschaftler nach Strategien. „Eine Möglichkeit ist es, Beta-Zellen der Bauchspeicheldrüse vor dem Untergang zu bewahren", sagt Prof. Dr. Anette-Gabriele Ziegler. Sie ist Direktorin des Instituts für Diabetesforschung (IDF) am Helmholtz Zentrum München, Leiterin der Forschergruppe Diabetes an der Technischen Universität München und Mitglied im Deutschen Zentrum für Diabetesforschung (DZD). In einer gemeinsamen Studie mit der Yale University New Haven konnten Ziegler und Kollegen zeigen, dass die Zerstörung der Betazellen durch das körpereigene Immunsystem mithilfe von Teplizumab gemindert werden kann. In der randomisierten, Placebo-kontrollierten doppelblinden Phase II-Studie verzögerte eine 14-tägige intravenöse Gabe von Teplizumab bei Personen mit einem hohen Typ-1-Diabetes-Risiko den Ausbruch der Autoimmunerkrankung im Durchschnitt um 2 Jahre.

\section{Neue Studie PROTECT}

In der neuen klinischen Forschungsstudie PROTECT wollen Forschende nun untersuchen, ob das Prüfmedikament Teplizumab auch bei Kindern und Jugendlichen im Alter von acht bis 17 Jahren mit einem kürzlich diagnostizierten Typ-1-Diabetes den Verlust von Betazellen verlangsamen kann. An der internationalen Studie beteiligen sich auch DZD-Wissenschaftlerinnen und Wissenschaftler des Universitätsklinikums Carl Gustav Carus, des Paul-LangerhansInstitutes Dresden des Helmholtz Zentrum München und der Medizinischen Fakultät der TU Dresden. Insgesamt sollen 300 Kinder und Jugendliche an Studienzentren in den USA, Kanada und Europa an der randomisierten, Placebo-kontrollierten doppelblinden Phase III Studie untersucht werden.

\section{Aufbau der Studie}

Die Teilnehmenden erhalten über eine intravenöse Infusion entweder das Prüfmedikament oder ein Placebo. Über einen Zeitraum von 12 Tagen bekommen sie täglich die jeweilige Infusion. Die Studie besteht aus zwei Zyklen. Nach 6 Monaten erhalten die Probanden daher erneut täglich über einen Zeitraum von 12 Tagen eine Infusion. Nach 18 Monaten untersuchen die Forschenden u.a. anhand vom $\mathrm{HbA} 1 \mathrm{c}$-Wert, Blutglukosewerte und der benötigen Menge an künstlichen Insulin, wie das Prüfmedikament wirkt. „Mit der Studie wollen wir herausfinden, ob die zweimalige Gabe von Teplizumab bei Kindern und Jugendlichen mit neudiagnostizierten Typ-1-Diabetes, die Zerstörung der Betazellen mildern kann und hilft, die Insulin-Produktion länger aufrecht zu erhalten", erläutert Prof. Dr. Reinhard Berner, PI der Studie und Direktor der Klinik und Poliklinik für Kinder- und Jugendmedizin am Uniklinikum Dresden. 